\title{
Evaluación del lavado gástrico como herramienta para el análisis de la dieta en trucha común
}

\author{
Javier Sánchez-Hernández ${ }^{1,3, *}$, María J. Servia ${ }^{2}$, Rufino Vieira-Lanero ${ }^{3}$ y Fernando Cobo ${ }^{1,3}$
}

${ }^{1}$ Departamento de Zoología y Antropología Física. Facultad de Biología. Universidad de Santiago de Compostela. 15782-Santiago de Compostela. España.

2 Departamento de Biología Animal, Biología Vegetal y Ecología. Facultad de Ciencias. Campus da Zapateira s/n. Universidad de A Coruña. 15008-A Coruña. España.

${ }^{3}$ Estación de Hidrobiología "Encoro do Con". Castroagudín-Cea. 36617-Vilagarcía de Arousa. Pontevedra. España.

* Autor responsable de la correspondencia: javier.sanchez@usc.es

Recibido: $1 / 2 / 10$

Aceptado: 18/6/10

\begin{abstract}
Evaluation of the gastric lavage as a tool for the analysis of the brown trout diet

The efficacy of the method of extracting stomach content, by means of the procedure of stomach washing through a pump, based on Seaburg's original model (1957), has been studied. The effectiveness of the method is not related to the size of the fish, but rather to the prey's own morphological characteristics, the degree of repletion of the stomach and the extent of digestion of the food. Thus, the effectiveness of the method is inversely related to the degree of repletion. Furthermore, bigger prey having rigid structures, such as Aeshnidae and Caddisflies with cases are more difficult to expel. However, the observed differences in the effectiveness of expulsion between preys with similar characteristics are due to the extent of their digestion. Nevertheless, the average effectiveness of the method is high and in excess of $80 \%$. It is therefore a relatively simple and effective methodology and the reason why its use is appropriate for obtaining stomach content samples in young and adult brown trout.
\end{abstract}

Key words: Salmo trutta, gastric lavage, stomach pump, effectiveness.

\section{RESUMEN}

\section{Evaluación del lavado gástrico como herramienta para el análisis de la dieta en trucha común}

Se ha estudiado la eficacia del método de extracción del contenido estomacal mediante el procedimiento de lavado estomacal a través de una bomba diseñada a partir del modelo original de Seaburg (1957). La efectividad del método no esta relacionada con el tamaño del pez, sino más bien con las propias características morfológicas de las presas, el estado de repleción del estómago y el grado de digestión del alimento. Así, la efectividad del método está inversamente relacionada con el estado de repleción. Además, presas de gran tamaño y que presentan estructuras rígidas como los Aeshnidae y los Tricópteros con estuches son más difíciles de expulsar. No obstante, las diferencias observadas en la efectividad de expulsión entre presas con las mismas características se deben al diferente grado de digestión de las mismas. Aún así, la efectividad media del método es elevada y superior al $80 \%$. Se trata pues de una metodología relativamente sencilla y eficaz, por lo que su uso es adecuado para la obtención de muestras de contenido estomacal en juveniles y adultos de trucha común.

Palabras clave: Salmo trutta, lavado gástrico, bomba estomacal, efectividad. 


\section{INTRODUCCIÓN}

El principal inconveniente de los estudios de alimentación en peces es que los ejemplares suelen ser sacrificados, y en los casos en que es necesaria la recogida de una gran cantidad de muestras, el estudio puede comprometer la supervivencia de la población a estudiar. Así, existe una larga tradición en el diseño de métodos alternativos al sacrificio para evitar la muerte de los peces, e intentar mantener la integridad de la muestra. Estos métodos incluyen la inserción de tubos que facilitan la salida del alimento al ejercer presión sobre el estómago (Forney, 1974), el uso de eméticos (Jernejcic, 1969), la succión directa del contenido estomacal (Robertson, 1945), el uso de gastroscopios (Van Den Avyle \& Roussel, 1980), el uso de fórceps (Wales, 1962), el lavado intestinal en especies herbívoras (Baker \& Fraser, 1976) y el lavado estomacal mediante el bombeo de agua (Seaburg, 1957; Meehan \& Miller, 1978; Culp et al., 1988; Lepak et al., 2006; Bogacka-Kapusta et al., 2007). Líneas de investigación más recientes utilizan el análisis de isótopos estables para integrar una imagen de las dietas a una escala temporal y espacial (Power et al., 2002; Cucherousset et al., 2007).

La extracción del contenido estomacal por medio de la bomba de Seaburg (1957) parece no tener efectos a largo plazo en la alimentación, condición y comportamiento de los peces (Meehan \& Miller, 1978; Strange \& Kennedy, 1981; Twomey \& Giller, 1990) y tiene un reducido efecto en la supervivencia de salmónidos (Meehan \& Miller, 1978; Strange \& Kennedy, 1981). Los test realizados para comprobar la validez del método, para lo cual los estómagos son diseccionados después del bombeo, indican que el procedimiento es altamente efectivo (Meehan \& Miller, 1978; Strange \& Kennedy, 1981; Lepak et al., 2006; Wanner, 2006; Bogacka-Kapusta et al., 2007). De entre todos los métodos no letales, el bombeo a presión mecanizado parece ser el método más eficaz para la mayoría de los ejemplares grandes, mientras que el uso de jeringuillas permite obtener buenos resultados en peces jóvenes y pequeños (Culp et al., 1988; Kamler \& Pope, 2001). Así, Strange \& Kennedy (1981) consideran que los ejemplares de tamaño inferio- res a $4 \mathrm{~cm}$ son demasiado pequeños para extraer el contenido estomacal con un chorro de agua, mientras que Hartleb \& Moring (1995) afirman que el tamaño del pez a partir del cual la extracción del alimento es efectiva es de $14 \mathrm{~cm}$.

Debido a su simplicidad y eficacia, el lavado estomacal se ha evaluado en numerosas especies, entre ellas muchos salmónidos (Neveu \& Thibault, 1977; Meehan \& Miller, 1978; Strange \& Kennedy, 1981; Culp et al., 1988; Lepak et al., 2006), y, a pesar de la escasez de trabajos similares de validación en la bibliografía reciente (Lepak et al., 2006; Bogacka-Kapusta et al., 2007), su uso está muy extendido. Sin embargo, la mayoría de los trabajos han estudiado la efectividad de la técnica con relación a las características del pez, y son extraordinariamente escasos los que tienen como objetivo el estudio de la efectividad de la bomba en función de otras variables de gran importancia como la naturaleza de las presas (tipo de taxón, tamaño de la presa o presencia de estructuras rígidas que puedan dificultar su expulsión) y el estado de saciedad del individuo. Por ello, la finalidad de este trabajo, más allá de validar la efectividad del método como han hecho otros autores, es comprobar si las variables antes mencionadas (tipo de taxón, tamaño y naturaleza de las presas y el estado de saciedad del pez) pueden limitar la efectividad del método.

\section{MATERIAL Y MÉTODOS}

Se ha comprobado la eficacia del lavado con la bomba estomacal diseñada a partir del modelo original de Seaburg (1957) en 66 individuos de cuatro ríos diferentes de Galicia (NW España). En el río Anllóns (UTM: 29T 509334 4786497) se analizaron 19 estómagos, en el río Furelos 11 (UTM: 29T 580203 4747203), en el río Lengüelle 18 (UTM: 29T 543882 4758938), y en el río Tambre 18 (UTM: 29T 556103 4760391). La toma de muestras en cuatro ríos distintos se hizo con la intención de que suministrasen, a prio$r i$, el espectro más amplio posible de diferencias alimentarias, y de esta manera comprobar la efectividad de este método para una mayor variedad de presas, ya que las características de la comuni- 
dad bentónica que presentan los ríos muestreados son diferentes (Sánchez-Hernández, 2009).

Los ejemplares de trucha común fueron capturados en septiembre de 2007 usando un equipo de pesca eléctrica (Hans Grassl GmbH, ELT60II). Inmediatamente después de la captura, los peces fueron anestesiados con benzocaína, y se recogieron los datos biométricos de longitud furcal (con una precisión de $1 \mathrm{~mm}$ ) y de peso (con una precisión de $0.1 \mathrm{~g}$ ). La edad fue determinada a partir de las escamas recogidas en todos los especímenes y el tamaño de las truchas estuvo comprendido entre 10.9 y $34.3 \mathrm{~cm}$.

Las muestras del contenido estomacal fueron recogidas en el campo mediante el lavado del estómago con la bomba. Los restos estomacales retenidos en la boca fueron retirados fácilmente con pinzas. El contenido de los estómagos fue recogido en botes de PVC y conservado en formaldehído al $10 \%$ para su posterior identificación. Una vez extraído el contenido estomacal, se sacrificaron los ejemplares para comprobar el vaciado del contenido estomacal y la efectividad de esta técnica.

En el laboratorio, se procedió a la disección del estómago y a la identificación de las presas bajo la lupa binocular. El estado de repleción $(f)$ fue calculado para cada ejemplar, $f=\left(W_{s} / W\right)_{*} 100$, donde $W_{s}$ es el peso del contenido estomacal (suma del peso de la proporción extraída en el campo y el peso de la proporción extraída en el laboratorio) y $W$ es el peso húmedo del pez, expresados en gramos. Para reducir al máximo el error en la estimación de la eficacia y del peso total, la muestra que se obtuvo en el campo (fijada en formaldehido) se filtró y se secó con papel de filtro. La longitud máxima de las presas $( \pm 0.01 \mathrm{~mm})$, incluyendo los cercos, se estimó en cada presa con ayuda de un micrómetro digital. La eficacia del método se evaluó como el porcentaje del alimento extraído respecto al total contenido en el estómago en términos de peso húmedo $(\mathrm{g})$ y número de presas.

Para el análisis estadístico de los datos se utilizó el paquete estadístico SPSS (Statistical Package for the Social Sciences, versión 15.0). Se realizaron análisis de varianza de dos vías para comparar las medias de las variables, aceptan- do que todas estas pruebas eran estadísticamente significativas siempre que la probabilidad asociada resultara ser menor que 0.05 . Para el análisis de las diferencias en función del sexo no se tuvieron en cuenta los ejemplares en los que no se pudo determinar éste, realizándose los test en 58 ejemplares. En el análisis por clases de edad no se contabilizó el único ejemplar 4+ capturado.

\section{RESULTADOS}

Todas las truchas analizadas presentaron alimento en su estómago. La disección de los estómagos demostró que la eficacia de este método es elevada, aunque en muchos casos no permite extraer todo el contenido estomacal. Así, la efectividad media de esta técnica, en términos de peso, fue del $80.49 \% \pm 3.918$. No hemos encontrado diferencias de efectividad entre los ríos, clases de edad ni sexos (ANOVA test $F=0.390 ;$ g.l. $=65$; $P=0.767$, ANOVA test $F=0.567 ;$ g.l. $=64$; $P=0.595$, ANOVA test $F=0.481 ;$ g.l. $=57$; $P=0.519$ respectivamente). La extracción con este método fue inferior al $50 \%$ del contenido estomacal únicamente en el $16.66 \%$ de la muestra. De hecho, en ciertos ejemplares la efectividad fue muy baja o nula, ya que en seis individuos el contenido estomacal extraído fue inferior al $10 \%$, no siendo ejemplares de pequeño tamaño, sino truchas con tamaños comprendidos entre los 12.8 y $22.9 \mathrm{~cm}$. Sin embargo, la eficacia del método, aunque no está correlacionada con la talla del pez $(r=0.032 ; P=0.796)$, disminuye con el estado de repleción del estómago $(r=-0.40 ; P=0.01)$.

La eficacia respecto al número de presas, al igual que ocurre con el valor porcentual del peso, es elevada y del $80.52 \% \pm 6.826$. Nuevamente no hemos encontrado diferencias significativas entre clases de edad ni sexos (ANOVA test $F=0.708$; g.l. $=64 ; P=0.497$, ANOVA test $F=0.171$; g.l. $=57 ; P=0.843$ respectivamente), aunque si entre los ríos (ANOVA test $F=3.054 ;$ g.l. $=65$; $P=0.035)$, habiendo encontrado la eficacia más alta en el río Anllóns $(88.30 \% \pm 4.101)$ y la menor en el río Furelos $(53.91 \% \pm 10.210)$. En la Tabla 1 se muestra la efectividad de este método en función de los ríos, las cohortes y los sexos 
Tabla 1. Datos biométricos (talla), estado de repleción, talla media de presa y porcentaje de alimento extraído por la bomba estomacal (media \pm error típico de la media). Biometric data (size), repletion state, mean size of prey and percentage of food extracted by the stomach pump (mean \pm S.E.).

\begin{tabular}{|c|c|c|c|c|c|c|c|}
\hline & & $\begin{array}{l}\text { Talla } \\
(\mathrm{cm})\end{array}$ & $\begin{array}{c}\text { Estado de } \\
\text { repleción }(\%)\end{array}$ & $\begin{array}{c}\text { Talla } \\
\text { presa }(\mathrm{mm})\end{array}$ & $\begin{array}{c}\text { Eficacia } \\
(\text { peso, \%) }\end{array}$ & $\begin{array}{c}\text { Eficacia } \\
\left(\mathrm{N}^{\circ} \text { presas, \%) }\right.\end{array}$ & $\begin{array}{c}\text { Tamaño de la } \\
\text { muestra }(n)\end{array}$ \\
\hline \multirow{4}{*}{ Ríos } & Anllóns & $20.9 \pm 1.34$ & $0.35 \pm 0.0877$ & $7.7 \pm 0.32$ & $88.3 \pm 5.21$ & $88.3 \pm 4.11$ & 19 \\
\hline & Furelos & $22.3 \pm 1.83$ & $1.02 \pm 0.123$ & $13.8 \pm 2.06$ & $63.6 \pm 10.01$ & $53.9 \pm 10.21$ & 11 \\
\hline & Lengüelle & $21.2 \pm 0.70$ & $0.52 \pm 0.184$ & $10.6 \pm 0.47$ & $84.9 \pm 7.31$ & $86.9 \pm 6.62$ & 18 \\
\hline & Tambre & $15.9 \pm 0.68$ & $0.44 \pm 0.073$ & $8.2 \pm 0.57$ & $78.1 \pm 8.9$ & $77.7 \pm 8.94$ & 18 \\
\hline \multirow{4}{*}{ Cohortes } & $1+$ & $14.8 \pm 0.33$ & $0.66 \pm 0.832$ & $8.7 \pm 0.62$ & $77.2 \pm 7.02$ & $75.3 \pm 6.93$ & 23 \\
\hline & $2+$ & $20.2 \pm 0.35$ & $0.48 \pm 0.123$ & $9.62 \pm 0.57$ & $80.8 \pm 6.19$ & $81.7 \pm 6.07$ & 29 \\
\hline & $3+$ & $27.1 \pm 0.45$ & $0.37 \pm 0.135$ & $11.5 \pm 1.73$ & $84.1 \pm 7.62$ & $87.8 \pm 5.21$ & 13 \\
\hline & $4+$ & 34.3 & 1.36 & 7.5 & 100 & 100 & 1 \\
\hline \multirow{3}{*}{ Sexo } & Macho & $22.1 \pm 0.85$ & $0.46 \pm 0.816$ & $9.6 \pm 0.72$ & $82 \pm 5.18$ & $80.8 \pm 5.37$ & 35 \\
\hline & Hembra & $18.3 \pm 0.83$ & $0.54 \pm 0.142$ & $9.9 \pm 0.72$ & $74.6 \pm 7.69$ & $78.3 \pm 6.96$ & 23 \\
\hline & Sin determinar & $14.7 \pm 0.41$ & $0.84 \pm 0.101$ & $9 \pm 1.33$ & $90.9 \pm 6.29$ & $85.68 \pm 8.45$ & 8 \\
\hline
\end{tabular}

respecto al peso del contenido estomacal respecto al número de presas.

El tamaño medio de las presas consumidas por el conjunto de la muestra fue $9.6 \mathrm{~mm} \pm 0.48$, estando comprendida entre los $5.3 \mathrm{~mm}$ y los $25.9 \mathrm{~mm}$. No hemos encontrado diferencias estadísticamente significativas entre clases de edad ni entre sexos en cuanto a la talla media de las presas (ANOVA test $F=1.53 ;$ g.l. $=64$; $P=0.216$, ANOVA test $F=0.146 ; g . l .=57$; $P=0.865$ respectivamente).
Desde el punto de vista cualitativo, la eficacia del método es aceptablemente buena, y siempre superior al $50 \%$ en todos los taxones presentes en los estómagos. Así, en 70 de los 86 $(81.4 \%)$ taxones identificados la efectividad es superior al $80 \%$, y en 51 familias $(59.3 \%$ ) es del $100 \%$, aunque la presencia de éstos últimos items en los estómagos es baja (Tabla 2). En la figura 1 se puede observar la eficacia del método en función los taxones identificados, siendo los de mayor tamaño los que presentaron las me-

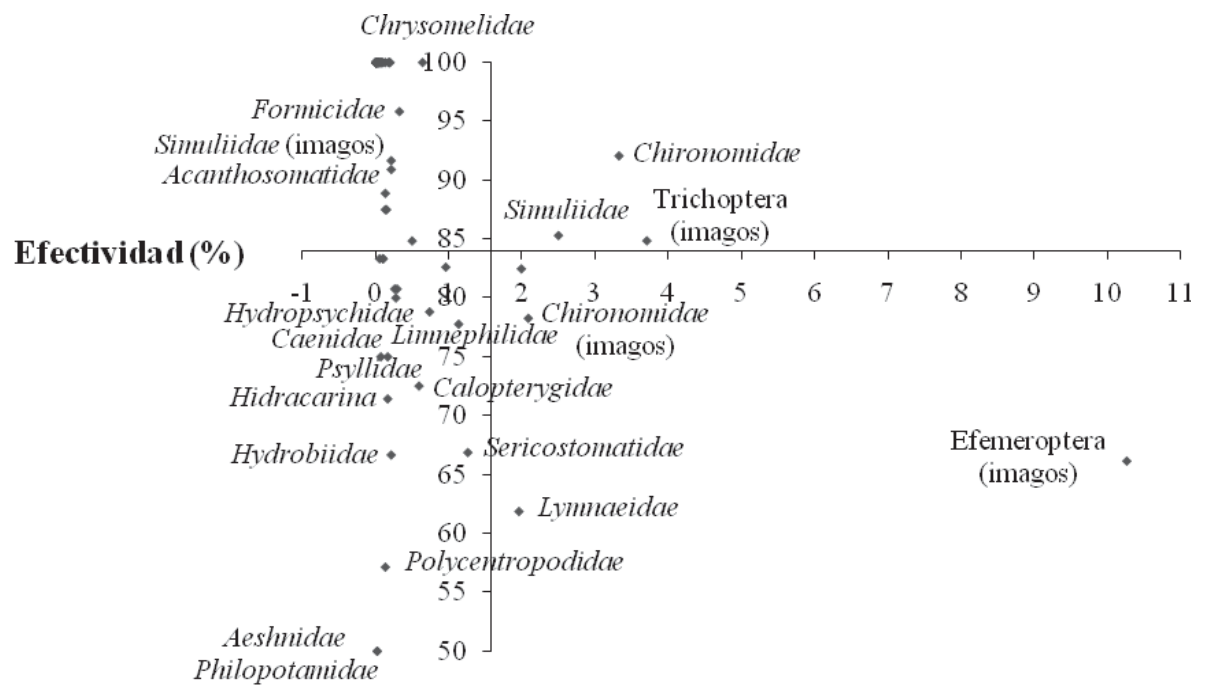

\section{$\mathrm{N}^{\circ}$ medio de ejemplares por trucha}

Figura 1. Eficacia del método según el tipo presa. El punto de corte de los ejes se corresponde con la media ponderada de cada variable. Efficacy of the method according to the type of prey. The point where the axes cross corresponds with the weighted average of each variable. 
Tabla 2. Eficacia total del método (en términos de número de presas) y por clases de edad. Sin identificar (s.i). Total Efficacy of the method (in terms of the number of preys) and by age class. Without identifying (s.i).

\begin{tabular}{|c|c|c|c|c|c|c|c|}
\hline & \multicolumn{2}{|c|}{$\mathrm{N}^{0}$ medio de presas/trucha } & \multicolumn{5}{|c|}{ Eficacia del método ( \%) } \\
\hline & Bomba & Totales & $1+$ & $2+$ & $3+$ & $4+$ & Total \\
\hline \multicolumn{8}{|c|}{ Invertebrados acuáticos } \\
\hline Erpobdellidae & 0.02 & 0.02 & - & 100 & - & - & 100 \\
\hline Ancylidae & 0.10 & 0.10 & 100 & 100 & - & - & 100 \\
\hline Hydrobiidae & 0.97 & 1.27 & 60 & 68.7 & 72 & - & 66.9 \\
\hline Lymnaeidae & 1.25 & 1.97 & 69.2 & 80.2 & 36.3 & - & 61.9 \\
\hline Sphaeriidae & 0.14 & 0.14 & 100 & 100 & 100 & - & 100 \\
\hline Hidracarina (s.i) & 0.13 & 0.17 & 71.4 & - & - & & 71.4 \\
\hline Gammaridae & 0.05 & 0.05 & 100 & 100 & - & - & 100 \\
\hline Ostracoda (s.i) & 0.05 & 0.05 & 100 & - & - & - & 100 \\
\hline Baetidae & 1.37 & 2.0 & 57 & 90.5 & 100 & - & 82.5 \\
\hline Caenidae & 0.05 & 0.08 & 25 & 100 & 100 & - & 75 \\
\hline Ephemerellidae & 0.10 & 0.10 & 100 & 100 & - & - & 100 \\
\hline Heptageniidae & 0.11 & 0.14 & 100 & 75 & - & - & 87.5 \\
\hline Leuctridae & 0.24 & 0.29 & 60 & 80 & 100 & - & 80 \\
\hline Perlidae & 0.02 & 0.02 & - & 100 & - & - & 100 \\
\hline Aeshnidae & 0.02 & 0.03 & 0 & 100 & - & - & 50 \\
\hline Calopterygidae & 0.30 & 0.60 & 60 & 85 & - & - & 72.5 \\
\hline Cordulegasteridae & 0.03 & 0.03 & 100 & - & 100 & - & 100 \\
\hline Gomphidae & 0.02 & 0.02 & 100 & - & - & - & 100 \\
\hline Platycnemididae & 0.02 & 0.02 & - & 100 & - & - & 100 \\
\hline Aphelocheiridae & 0.46 & 0.97 & 93.7 & 54.1 & 100 & - & 82.6 \\
\hline Gerridae & 0.21 & 0.22 & 100 & 100 & 72.7 & - & 90.9 \\
\hline Hydrometridae & 0.02 & 0.02 & - & - & 100 & - & 100 \\
\hline Nepidae & 0.06 & 0.06 & 100 & - & 100 & - & 100 \\
\hline Sialidae & 0.03 & 0.03 & 100 & - & - & - & 100 \\
\hline Dytiscidae & 0.08 & 0.08 & - & 100 & - & 100 & 100 \\
\hline Elmidae & 0.10 & 0.11 & 66.6 & 100 & - & - & 83.3 \\
\hline Gyrinidae & 0.02 & 0.02 & - & 100 & - & - & 100 \\
\hline Hydrophilidae & 0.02 & 0.02 & 100 & - & - & - & 100 \\
\hline Brachycentridae & 0.03 & 0.03 & - & 100 & - & - & 100 \\
\hline Goeridae & 0.03 & 0.03 & - & 100 & 100 & - & 100 \\
\hline Hydropsychidae & 0.63 & 0.75 & 63 & 73.4 & 100 & - & 78.8 \\
\hline Lepidostomatidae & 0.02 & 0.02 & - & - & 100 & - & 100 \\
\hline Leptoceridae & 0.02 & 0.02 & 100 & 100 & - & - & 100 \\
\hline Limnephilidae & 0.71 & 1.14 & 54.8 & 56.4 & 100 & 100 & 77.8 \\
\hline Philopotamidae & 0.02 & 0.03 & - & 50 & - & - & 50 \\
\hline Polycentropodidae & 0.06 & 0.14 & 81.3 & 90 & 0 & - & 57.1 \\
\hline Psychomyiidae & 0.14 & 0.16 & 100 & 62.5 & 100 & - & 87.5 \\
\hline Rhyacophilidae & 0.25 & 0.30 & 61.7 & 80.7 & 100 & - & 80.8 \\
\hline Sericostomatidae & 0.16 & 0.22 & 34.9 & 65.2 & 100 & - & 66.7 \\
\hline Chironomidae & 3.08 & 3.33 & 91 & 91.2 & 94.1 & - & 92.1 \\
\hline Empididae & 0.19 & 0.19 & 100 & 100 & - & - & 100 \\
\hline Limoniidae & 0.02 & 0.02 & - & 100 & - & - & 100 \\
\hline Simuliidae & 2.24 & 2.51 & 85.9 & 70 & 100 & - & 85.3 \\
\hline
\end{tabular}


Tabla 2. cont.

\begin{tabular}{|c|c|c|c|c|c|c|c|}
\hline & \multicolumn{3}{|c|}{$\mathrm{N}^{0}$ medio de presas/trucha } & \multicolumn{4}{|c|}{ Eficacia del método ( \%) } \\
\hline & Bomba & Totales & $1+$ & $2+$ & $3+$ & $4+$ & Total \\
\hline \multicolumn{8}{|l|}{ Imagos acuáticos } \\
\hline Efemeroptera (s.i) & 6.79 & 10.27 & 33.5 & 64.8 & 100 & - & 66.1 \\
\hline Odonata (s.i) & 0.02 & 0.02 & - & 100 & - & - & 100 \\
\hline Leptoceridae & 0.06 & 0.06 & 100 & - & - & - & 100 \\
\hline Trichoptera (s.i) & 3.59 & 3.71 & 64.2 & 90.5 & 100 & - & 84.9 \\
\hline Chironomidae & 1.87 & 2.10 & 62.9 & 72 & 100 & - & 78.3 \\
\hline Empididae & 0.19 & 0.19 & 100 & 100 & - & - & 100 \\
\hline Simuliidae & 0.21 & 0.22 & 100 & 100 & 75.1 & - & 91.7 \\
\hline \multicolumn{8}{|l|}{ Invertebrados terrestres } \\
\hline Araneidos (s.i) & 0.11 & 0.11 & 100 & 100 & 100 & - & 100 \\
\hline Orthoptera (s.i) & 0.02 & 0.02 & - & - & 100 & - & 100 \\
\hline Forficulidae & 0.02 & 0.02 & - & - & 100 & - & 100 \\
\hline Acanthosomatidae & 0.13 & 0.14 & 100 & 66.7 & 100 & - & 88.9 \\
\hline Heteroptera (s.i) & 0.10 & 0.11 & 100 & 49.9 & 100 & - & 83.3 \\
\hline Lygaeidae & 0.03 & 0.03 & 100 & - & - & - & 100 \\
\hline Reduviidae & 0.08 & 0.08 & 100 & 100 & 100 & - & 100 \\
\hline Tingidae & 0.02 & 0.02 & - & 100 & - & - & 100 \\
\hline Aphididae & 0.03 & 0.03 & - & - & 100 & - & 100 \\
\hline Cicadellidae & 0.21 & 0.21 & 100 & 100 & 100 & - & 100 \\
\hline Psyllidae & 0.13 & 0.17 & 100 & 25 & 100 & - & 75 \\
\hline Lepidoptera (Larva) (s.i) & 0.02 & 0.02 & - & 100 & - & - & 100 \\
\hline Thysanoptera (s.i) & 0.02 & 0.02 & 100 & - & - & - & 100 \\
\hline Acanthosomidae & 0.02 & 0.02 & - & - & 100 & - & 100 \\
\hline Calliphoridae & 0.02 & 0.02 & 100 & - & - & - & 100 \\
\hline Camillidae & 0.02 & 0.02 & - & - & 100 & - & 100 \\
\hline Diptera (s.i) & 0.44 & 0.51 & 100 & 71.1 & 83.3 & - & 84.8 \\
\hline Dolichopodidae & 0.02 & 0.02 & - & 100 & - & - & 100 \\
\hline Musidae & 0.05 & 0.05 & - & 100 & 100 & - & 100 \\
\hline Tipulidae & 0.02 & 0.02 & - & 100 & 一 & - & 100 \\
\hline Xylomyidae & 0.02 & 0.02 & 100 & - & - & - & 100 \\
\hline Apoidea & 0.02 & 0.02 & - & - & 100 & - & 100 \\
\hline Cynipidae & 0.05 & 0.05 & - & 100 & 100 & - & 100 \\
\hline Formicidae & 0.32 & 0.33 & 100 & 87.4 & 100 & - & 95.8 \\
\hline Hymenoptera (s.i) & 0.02 & 0.02 & 100 & - & - & - & 100 \\
\hline Ichneumonidae & 0.02 & 0.02 & - & 100 & - & - & 100 \\
\hline Sphecidae & 0.02 & 0.02 & 100 & - & - & - & 100 \\
\hline Chrysomelidae & 0.65 & 0.65 & 100 & 100 & 100 & - & 100 \\
\hline Coccinellidae & 0.03 & 0.03 & 100 & 一 & - & - & 100 \\
\hline Coleoptera (s.i) & 0.21 & 0.27 & 100 & 80 & 62.1 & - & 80.7 \\
\hline Curculionidae & 0.05 & 0.06 & 100 & 66.6 & - & - & 83.3 \\
\hline Oligochaeta (s.i) & 0.02 & 0.02 & 100 & - & - & - & 100 \\
\hline Panorpidae & 0.02 & 0.02 & 100 & - & - & - & 100 \\
\hline \multicolumn{8}{|l|}{ Otras presas } \\
\hline Pseudochondrostoma duriense & 0.22 & 0.22 & 100 & 100 & 100 & - & 100 \\
\hline Huevo Pseudochondrostoma duriense & 0.16 & 0.16 & 100 & 100 & 100 & - & 100 \\
\hline
\end{tabular}


nores tasas de efectividad, como es el caso de Aeshnidae $($ media $=22.17 \mathrm{~mm}$; rango de tamaño: $15-33 \mathrm{~mm}$ ), Philopotamidae $($ media $=9.5 \mathrm{~mm}$; rango de tamaño: 8-12 mm) y Polycentropodidae (media $=9.83 \mathrm{~mm}$; rango de tamaño: 9-12 mm). Por el contrario presas muy pequeñas, de un tamaño inferior a $5 \mathrm{~mm}$, como por ejemplo Ostracoda, Cynipidae, Gyrinidae y Sphaeriidae), y en un elevado estado de digestión (como por ejemplo: Hydrometridae, Acanthosomidae, Cordulegasteridae, Dytiscidae y Aphelocheiridae) fueron expulsadas efectivamente.

\section{DISCUSIÓN}

Se ha apuntado, en algunas ocasiones, que la utilización de la pesca eléctrica como método de captura está asociada a la regurgitación del contenido estomacal (García de Jalón et al., 1993). Aún así, el objetivo primordial de este trabajo justifica la utilización de esta técnica, pues independientemente de su cantidad original, se comparan la naturaleza de las presas en el estómago antes y después de su lavado por medio de la bomba.

La extracción del contenido estomacal por medio de la bomba diseñada por Seaburg (1957) es una técnica recomendada si se quiere devolver con vida al animal y reducir al mínimo el efecto del muestreo sobre las poblaciones (Meehan \& Miller, 1978; Twomey \& Giller, 1990). Además se ha empleado con éxito en estudios de alimentación (Lepak et al., 2006; Bogacka-Kapusta et al., 2007), de conversión del alimento (Swenson \& Smith, 1973) y de cálculo de tasas digestivas (Seaburg \& Moyle, 1964).

La mayoría de los test realizados para comprobar la validez de la bomba estomacal indican que el procedimiento es altamente efectivo (Meehan \& Miller, 1978; Lepak et al., 2006; Wanner, 2006; Bogacka-Kapusta et al., 2007). Así, Wanner (2006) obtuvo en juveniles de esturión (Scaphirhynchus albus) que la efectividad del método es semejante cuando se analiza el número de presas y el peso del contenido estomacal (74.9\% y $73.7 \%$ respectivamente).

Observaciones muy parecidas fueron realizadas por Bogacka-Kapusta et al. (2007) para juve- niles de otra especie de esturión (Acipenser ruthenus), ya que el lavado gástrico permite determinar el $65 \%$ de la dieta. Por el contrario Lepak et al. (2006) encontraron en salvelino (Salvelinus namaycush) valores superiores y con una efectividad del método del $90 \%$. En nuestro caso, en la mayoría de las truchas analizadas (69.70\%) los resultados obtenidos se asemejan a los de estos autores, y la efectividad del método es elevada y superior al $80 \%$. Además se da la circunstancia de que la efectividad media del método es semejante cuando se evalúa el método de acuerdo al peso o el número de presas, como en su día observó Wanner (2006).

Neveu \& Thibault (1977) encontraron que la efectividad del método, a pesar de ser buena, es incierta ya que depende del tamaño del estómago y de los grupos faunísticos encontrados en el estómago. De hecho, a pesar de ser una metodología efectiva, hemos encontrado que la efectividad del método está inversamente relacionada con el estado de repleción del estómago. Esto podría deberse a que en las truchas con elevadas cantidades de alimento en el estómago la masa alimenticia está más apelmazada, aspecto que dificulta su expulsión.

En cuanto a los grupos faunísticos, Neveu \& Thibault (1977) encontraron que los Efemerópteros, Dípteros y Coleópteros eran eficazmente expulsados, y que las presas de pequeño tamaño eran más fáciles de expulsar que las grandes. En nuestro caso, hemos observado que la eficacia es variable aunque siempre superior al $50 \%$ en todos los taxones presentes en los estómagos. Además, si tenemos presente que las presas más abundantes en la alimentación de la trucha común en la mayoría de los estudios efectuados en la Península Ibérica son los Efemerópteros, Dípteros y Tricópteros (véase por ejemplo García de Jalón \& Barceló, 1987; Suárez et al., 1988; Rincón \& Lobón-Cerviá, 1999; Oscoz et al., 2000; Montori et al., 2006), la efectividad del método es elevada para la mayor parte de las familias de estos grupos de Insectos, como los Dípteros: Chironomidae y Simuliidae, los Efemerópteros: Baetidae, Ephemerellidae y Heptageniidae, y los Tricópteros: Hydropsychidae, Limnephilidae y Rhyacophilidae, lo cual nos 
permite concluir que presas con elevadas frecuencias de consumo por las truchas son bien expulsadas con este método.

Neveu \& Thibault (1977) y Kamler \& Pope (2001) concluyen que la efectividad de los métodos no letales, como el utilizado en el presente trabajo, dependen del tamaño de las presas presentes en el estómago, aunque en la bibliografía sobre el uso de la bomba en trucha común no hemos encontrado referencias que permitan corroborar esta afirmación. En nuestro trabajo hemos observado que presas grandes como los Aeshnidae son difíciles de expulsar, presentando la bomba una efectividad para ellos del $50 \%$. Sin embargo, presas de tamaños semejantes, pero que en nuestro caso aparecieron más fragmentadas, como los Cordulegasteridae, Gomphidae, Aphelocheiridae, Lepidostomatidae, Leptoceridae, Acanthosomatidae, Limnephilidae, Calopterygidae y Sericostomatidae, fueron eficazmente desalojadas. Por otro lado, restos muy fragmentados y de muy pequeño tamaño se retienen con mayor facilidad en el estómago, ya que pueden quedar retenidos entre los pliegues estomacales. De esta manera se podría justificar la presencia de numerosos fragmentos de imagos de Efemerópteros en muchos estómagos, ya que la mayoría de los ejemplares mostraron un elevado grado de digestión, y los restos recuperados fueron mayoritariamente fragmentos torácicos. Así pues los extremos en el tamaño del alimento que se encuentra en el estómago se desalojan con mayor dificultad por la acción del lavado.

Las presas que tienen estructuras rígidas, como es el caso de los Tricópteros con estuche, presentan una mayor resistencia a la expulsión (Sericostomatidae y Limnephilidae por ejemplo), aunque la diversidad de resultados obtenidos en nuestro caso puede deberse al diferente grado de digestión de cada presa, ya que en algunas el estuche aparece completamente disgregado, facilitándose así su expulsión. De igual manera, en los Gasterópodos, Lymnaeidae e Hydrobiidae, la efectividad obtenida no es muy elevada (alrededor del $65 \%$ ), probablemente debido al peso de la concha, que dificulta su expulsión.

Esta técnica no está exenta de críticas. La principal desventaja es el límite inferior de ta- maño de los peces a partir del cual es recomendable su utilización (Meehan \& Miller, 1978; Waters et al., 2004), ya que en peces muy pequeños no se puede poner en práctica esta técnica porque las propias características anatómicas del pez, como la apertura bucal, lo imposibilitan. Strange \& Kennedy (1981) consideran que ejemplares de tamaño inferior a $4 \mathrm{~cm}$ son demasiado pequeños para extraer el contenido estomacal con un chorro de agua, mientras que Hartleb \& Moring (1995) afirman que el tamaño del pez a partir del cual la extracción del alimento es efectiva es de $14 \mathrm{~cm}$. En nuestro caso, en función del tamaño de las truchas analizadas (todas mayores de $10 \mathrm{~cm}$ ), parece que el tamaño del pez no influye en la efectividad del método, sino más bien las características morfológicas de las presas (tamaño y presencia de estructuras rígidas), el estado de repleción del estómago y el grado de digestión de las presas.

Los resultados obtenidos nos permiten concluir que la efectividad de la extracción del contenido estomacal mediante este procedimiento, a partir de peces mayores de $10 \mathrm{~cm}$, es buena y no está relacionada con el tamaño del pez, sino con las características morfológicas de las presas, su grado de digestión y el estado de repleción del estómago. De esta forma se recomienda la recogida de muestras para los análisis dietéticos con el método evaluado en el presente trabajo, sobre todo si se va a trabajar con poblaciones en un estado crítico de conservación.

\section{AGRADECIMIENTOS}

Parte de este trabajo ha sido realizado en los laboratorios de la Estación de Hidrobiología de la USC "Encoro do Con" en Vilagarcía de Arousa. Este trabajo se ha realizado con el apoyo del proyecto INCITE09203072PR de la Xunta de Galicia. Los autores quieren agradecer a los dos evaluadores anónimos sus útiles comentarios y sugerencias.

\section{BIBLIOGRAFÍA}

BAKER, A. M. \& D. F. FRASER. 1976. A method for securing the gut contents of small, live fish. Trans. 
Am. Fish. Soc., 105: 520-522.

BOGACKA-KAPUSTA, E., A. KAPUSTA, A. DUDA, M. SZCZEPKOWSKI \& R. KOLMAN. 2007. Evaluation of the suitability of samples collected in vivo for investigations of juvenile sturgeon stomach contents. Arch. Pol. Fish., 15: 165-170.

CUCHEROUSSET, J., J. C. AYMES, F. SANTOUL \& R. CÉRÉGHINO. 2007. Stable isotope evidence of trophic interactions between introduced brook trout (Salvelinus fontinalis) and native brown trout (Salmo trutta) in a mountain stream of southwest France. J. Fish Biol., 71: 210-223.

CULP, J. M., I. BOYD \& N. E. GLOZIER. 1988. An improved method for obtaining gut contents from small, live fishes by anal and stomach flushing. $\mathrm{Co}$ peia, 4: 1079-1082.

FORNEY, J. L. 1974. Interactions between yellow perch abundance, walleye predation, and survival of alternate prey in Oneida Lake, New York. Trans. Am. Fish. Soc., 103: 15-24.

GARCÍA DE JALÓN, D. \& E. BARCELÓ. 1987. Estudio sobre la alimentación de la trucha común en los ríos Pirenaicos. Ecología, 1: 263-569.

GARCÍA DE JALÓN, D., M. MAYO, F. HERVELLA, E. BARCELÓ \& T. FERNÁNDEZ. 1993. Principios y técnicas de gestión de la pesca en aguas continentales. Mundi-Prensa Libros S.A. Madrid. 247 pp.

HARTLEB, C. F. \& J. R. MORING. 1995. An improved gastric lavage device for removing stomach contents from live fish. Fish. Res., 24: 261-265.

JERMEJCIC, F. 1969. Use of emetics to collect stomach contents of walleye and largemouth bass. Trans. Am. Fish. Soc., 98: 698-702.

KAMLER, J. K. \& K. L. POPE. 2001. Nonlethal methods of examining fish stomach contents. Rev. Fish. Sci., 9: 1-11.

LEPAK, J. M., C. E. KRAFT \& B. C. WEIDEL. 2006. Rapid food web recovery in response to removal of an introduced apex predator. Can. J. Fish. Aquat. Sci., 63: 569-575.

MEEHAN, W. R. \& R. A. MILLER. 1978. Stomach flushing: effectiveness and influence on survival and condition of juvenile salmonids. J. Fish. Res. Board Can., 35: 1359-1363.

MONTORI, A., J. M. TIERNO DE FIGUEROA \& X. SANTOS. 2006. The diet of the brown trout Salmo trutta (L.) during the reproductive period: sizerelated and sexual effects. Internat. Rev. Hydrobiol., 91: 438-450.
NEVEU, A. \& M. THIBAULT. 1977. Comportement alimentaire d'une population sauvage de truites fario (Salmo trutta L.) dans un ruisseau des Pyrénées Atlantiques, le Lissuraga. Ann. Hydobiol., 8: 111128.

OSCOZ, J., M. C. ESCALA \& F. CAMPOS. 2000. La alimentación de la trucha común (Salmo trutta L., 1758) en un río de Navarra (N. España). Limnetica, 18: 29-35.

POWER, M., G. POWER, F. CARON, R. R. DOUCETT \& K. R. GUIGUER. 2002. Growth and dietary niche in Salvelinus alpinus and Salvelinus fontinalis as revealed by isotope analysis. Env. Biol. Fish., 64: 75-85.

RINCÓN, P. A. \& J. LOBÓN-CERVIÁ. 1999. Preysize selection by brown trout (Salmo trutta L.) in a stream in northern Spain. Can. J. Zool., 77: 755765.

ROBERTSON, O. H. 1945. A method for securing stomach contents of live fish. Ecology, 26: 95-96.

SÁNCHEZ-HERNÁNDEZ, J. 2009. Biología de la alimentación de la trucha común (Salmo trutta Linné, 1758) en los ríos de Galicia. Tesis Doctoral. Universidad de Santiago de Compostela. 581 pp.

SEABURG, K. G. 1957. A stomach sampler for live fish. Prog. Fish-Cult., 19: 137-139.

SEABURG, K. G. \& J. B. MOYLE. 1964. Feeding habits, digestive rates and growth of some Minnesota warm-water fishes. Trans. Am. Fish. Soc., 93: 269-285.

STRANGE, C. D. \& G. J. KENNEDY. 1981. Stomach Flushing of Salmonids: A Simple and Effective Technique for the Removal of the Stomach Contents. Aquacult. Res., 12: 9-15.

SUÁREZ, J. L., L. REIRIZ \& R. ANADON. 1988. Feeding relationships between two salmonid species and the benthic community. Pol. Arch. Hydrobiol., 35: 341-351.

SWENSON, W. A. \& L. L. SMITH. 1973. Gastric digestion, food consumption, feeding periodicity and food conversion efficiency in walleye. J. Fish. Res. Bd. Can., 30: 1327-1336.

TWOMEY, R. H. \& P. S. GILLER. 1990. Stomach flushing and individual panjet tattooing of salmonidae: an evaluation of the long term effects on two wild populations. Aquacult. Fish. Manage., 21: 137-142.

VAN DEN AVYLE, M. J. \& J. E. ROUSSEL. 1980. Evaluation of a simple method for removing food items from live black bass. Prog. Fish-Cult., 42: 222-223. 
WALES, J. H. 1962. Forceps for removal of trout stomach content. Prog. Fish-Cult., 24: 171.

WANNER, G. A. 2006. Evaluation of a gastric lavage method on juvenile pallid sturgeon. N. Am. J. Fish. Manage., 26: 587-591.
WATERS, D. S., T. J. KWAK, J. B. ARNOTT \& W. E. PINE. 2004. Evaluation of stomach tubes and gastric lavage for sampling diets from blue catfish and flathead catfish. N. Am. J. Fish. Manage., 24: 258261. 